\title{
Acute $T$ cell-mediated rejection after administration of the BNT162b2 mRNA COVID-19 vaccine in a kidney transplant recipient without a history of acute rejection for 13 years: a case report
}

Hye-won Jang ${ }^{1}$, Sung Shin ${ }^{2}$

${ }^{1}$ Department of Surgery, Asan Medical Center, University of Ulsan College of Medicine, Seoul, Korea
${ }^{2}$ Department of Surgery-Transplantation, Asan Medical Center, University of Ulsan College of Medicine, Seoul, Korea

Background: Kidney transplant recipients have significantly high risks of mortality and morbidity after severe acute respiratory syndrome coronavirus 2 (SARS-CoV-2) infection, suggesting the need for earlier administration of coronavirus disease 2019 (COVID-19) vaccines in these individuals. However, there is limited data on the humoral and cellular responses after COVID-19 vaccination.

Case report: We report the case of a 78-year-old kidney transplant recipient who experienced acute $T$ cell-mediated rejection (TCMR) after receiving the second dose of the BNT162b2 mRNA COVID-19 vaccine (Pfizer-BioNTech). The recipient underwent deceased donor kidney transplantation for hypertension 13 years ago, and had not experienced any adverse event after transplantation. Her maintenance immunosuppressants were tacrolimus, azathioprine, and low-dose steroid. The latest level of serum creatinine 1 month before the vaccination was $0.61 \mathrm{mg} / \mathrm{dL}$ and the trough level of tacrolimus was maintained at $4-5 \mathrm{ng} / \mathrm{mL}$. Fifteen days after receiving the second dose of the BNT162b2 vaccine, the recipient visited our center with a mild headache and fever. The level of serum creatinine was elevated to $4.95 \mathrm{mg} / \mathrm{dL}$, and there was considerable swelling of the transplanted kidney on non-enhanced computerized tomography. On kidney biopsy, acute TCMR (grade IB) was diagnosed with no pathologic evidence of antibody-mediated rejection. Luminex single-antigen flow beads assay did not reveal donor-specific anti-HLA antibodies. AntiSARS-CoV-2 spike IgG and IgM antibodies (S1-IgG and S1-IgM) were measured by enzyme-linked immunosorbent assay (ELISA) on the day of the kidney biopsy (18 days after the second vaccination), which revealed that the levels of S1-IgG and S1-IgM were 2.80 (weak positive) and 0.16 (negative), respectively. The recipient was administered with steroid pulses (500 mg/day) for 5 days. One month after the steroid pulse therapy, her serum creatinine level had decreased to $2.47 \mathrm{mg} / \mathrm{mL}$.

Conclusions: This report shows that kidney transplant recipients may be at risk for acute rejection after COVID-19 vaccination despite having low levels of S1-IgG and S1-IgM. 